Medical Society. October, 1902); La Polymastio au Japon (Extract des Archives de Médecine Expérimentale et d'Anatomie Pathologique, fondées par J.-M. Charcot, et publiées par MM. Grancher. Joffroy, Lépine. No. 4, Juillet, 1904. Otani, S.: Beobachtungen über die Hypermastie (Mittheilungen der Medicinischen Gesellschaft zu Tokyo, Mai, 1899, Band xiii., No. 9); Nachtrag zur Hyperthelie (Ibid., Mai, 1902, Band xvi., No, 10). Sato, K.: Ein Fall von Regeneration der Polymastie nach einer Operation (Ibid., Oktober, 1904, Band xviii., No. 20). Polymastie et Tuberculosis, Extrait de No. 4 (La Presse Médicale, No. 82 , le 3e Octobre, 1906).

\section{THREE CASES OF LOBAR PNEUMONIA WITH UNUSUAL COMPLICATIONS.}

BY P. C. P. INGRAM, M.B., B.S.LOND.,

ASSISTANT MEDICAL OFFICER TO ST. GEORGE'S INFIRMARY, FULHAM-ROAD, S.W.

CASE 1.-The patient was a man, aged 22 years. His previous medical history was unimportant, but he had recently been drinking rather heavily. He was admitted to St. George's Infirmary on the second day of his illness. He showed the characteristic signs of commencing consolidation at the base of his left lung, with high temperature, dyspncea, and herpes on the lips. In addition, there was a patchy erythematous rash on the palmar and dorsal surfaces of the hands and plantar and dorsal surfaces of his feet. There were a similar area about two inches square on the left side of his face below the malar prominence and a few smaller ones on the abdomen and back. Except that for the first five days there was no expectoration, and then that it was white though viscid, the symptoms and physical signs were all those of a severe lobar pneumonia, and by the fifth day of his illness the signs of solid lung had extended upwards as far as the level of the scapular spine. The rash began to fade on the day after admission, first on the hands and feet, but on the fifth day of illness fresh patches appeared on the trunk. Two days later these were also much less distinct, but a few others more punctate in character appeared on the arms and legs. By this time the whole of the left lung except the apex of the upper lobe was involved; there were, however, no signs of pericarditis and but little of dilatation of the right side of the heart, but much restlessness and delirium. The temperature, hitherto varying from $100^{\circ}$ to $103^{\circ} \mathrm{F}$, now fell but did not reach normal for 24 hours, but signs of œdema of the right lung appeared and he died on the ninth day of illness. Permission to make a post-mortem examination could not be obtained.

CASE 2. - The patient was a man, aged 60 years. He had had fair health up to the commencement of the winter (six months previously), since when he had been troubled with cough and three months ago had an attack of "influenza" which kept him in bed for three weeks. Since then the cough had been worse and he had been losing llesh, but was able to get about. He was taken acutely ill the day previously to admission to St. George's Infirmary with dyspncea, tight cough, and blood-stained expectoration. On admission there were slight pyrexia $\left(99 \cdot 2^{\circ} \mathrm{F}\right.$.), dyspnoea, cough, and much rusty sputum. There was no herpes. The right lung was affected, showing dulness on percussion in front over the lower portion and behind over the mid-scapular area with a corresponding part in the axilla. Above the note was high pitched and below it was normal. Bronchial breathing and consonating râles were heard over the dull area. The left lung was clear except for a few scattered râles. The area of cardiac dulness was normal but the sounds were rapid and weak. On the following day the lung signs had spread a little upwards and in addition there were a patchy erythematous rash on the hands and wrists and a few faint patches on the legs. There was moderate pyrexia, the evening temperature reaching $100^{\circ}$. A diastolic bruit could now be heard in the aortic area. On the fourth day of illness the rash had faded considerably ; a pleural rub was now heard in the left axillary region. On the following day the patient died suddenly.

Necropsy.-The post-mortem examination showed a lobar pneumonia involving the upper lobe and upper half of the lower lobes of the right lung. At the apex of each lobe it had reached the stage of grey hepatisation, being less advanced in the intervening part of the lung. The rest of the right Iung was congested and the left lung was cedematous, with some recent plearisy over the middle of its lower lobe. There were scars of healed pulmonary tuberculosis at the apices of both upper lobes. The heart was enlarged, slightly fatty, and there was atheroma of the aorta involving one of the cusps of the aortic valves. The kidneys were slightly granular. The other organs were normal.

CASE 3 -The patient was a man, aged 36 years. His previous health was good. He had a rigor and pain in his left side the day before admittance to St. George's Infirmary. On admission his temperature was $103^{\circ} \mathrm{F}$; t there were signs of early. pneumonia as shown by dyspncea, cough, rusty sputum, and faint friction sound at the base of the left axilla, and on the following day definite signs of pneumonia as shown by bronchial breathing and consonating râles in that area. He was already severely ill on admission, the pulse being rapid and feeble and the urine contained a trace of albumin. The sputum examined two days after admission showed a fair number of pneumococci but no influenza or tubercle bacilli. The pneumonia was of a severe type with moderate pyrexia (the temperature varying from $99^{\circ} 0^{\circ}$ to $1024^{\circ}$ ) and much toxæmia. By the sixth day of illness bronchial breathing conld be heard up to the apex of the lung behind. On the seventh day the temperature fell by crisis with diarrhœe and sweating. Signs of cardiac dilatation became marked at the same time but the heart improved under treatment, strychnine being pushed to the extent of hypodermic injections of the liquor, five minims every four hours, in addition to moderate doses of digitalis and alcohol. On the day that the temperature reached normal a papular ery thematous rash appeared on the legs and back, being most marked on the knees, buttocks, and shoulders, and at the same time a purpuric rash in the left iliac fossa. The rash began to fade on the following day and was gone in another two days. By this time the lung showed signs of "clearing up" and convalescence appeared to be commencing. On the next day, however, there were some reappearance of pyrexia and signs of pneumonic infiltration at the opposite base. In two days the signs had spread up to the level of the spine of the scapula. Jaundice now appeared and by the next day was intense. The inflammation of the lung did not spread much further on this side of the chest and two days later showed signs of subsiding, the temperature never very high, falling by lysis and the jaundice diminishing at the same time. The urine no longer contained albumin, though the chlorides were still deficient. Three days later he developed a left-sided parotitis ; this lasted for some four days, the temperature going up to $103^{\circ}$ The lungs in the meantime were clearing but slowly, the right in particular still showing signs of consolidation over the base. At the end of a week from its commencement the parotitis was well, but two days previously to this he had complained of pain in the hypogastric region and slight tenderness on pressure was noticed there. At first no obvious cause could be found for this, the urine being normal and the bowels satisfactory, but two days later there was a definite sense of resistance on pressure being made a little above the pubes in the middle line. Nothing, however more definite appeared at the time. The temperature still remained irregular and there were impaired percussion note and diminished air entry at the base of the right lung, and he still had a cough with now a good deal of purulent sputum. The lung was accordingly explored by needle but no pus was found, and a few days later, his condition remaining unimproved, was again explored but without success. Meanwhile a painless swelling had been forming in the hypogastric region where previously had been felt that sense of resistance; it was exactly in the middle line, not more than two inches in diameter, without heat, redness, or tenderness. A couple of days after the time the lung was explored a second time the swelling began to increase more rapidly in size and then to fluctuate, so the skin having been anæsthetised by hypodermic injections of eucaine and adrenalin it was explored and found to consist of an abscess beneath and between the recti muscles. Several ounces of pus were let out and the cavity was drained. A culture of the pus gave pure pneumococci. After this the temperature began to settle and a week later it was normal. The lung signs slowly cleared up and a fortnight later the right was clear and a few râles only could be heard at the left base on deep breathing, and the patient was well enough to get up. A week later these had disappeared; he was up all day and gaining flesh and strength and went out to look for work some three weeks later.

These cases are recorded as examples of the rarer complications of lobar pneumonia. Each had an erythematous rash and Case 3 a purpuric rash as well. Case 3 had in addition parotitis and a pneumococcal abscess.

Except for the characteristic herpes of the lips skin lesions 
are uncommon in pneumonia. A purpuric rash does occasionally occur. Seven cases were seen and recorded by Thomas. ${ }^{1}$ Urticaria is still less common, but has been described by Traube ${ }^{2}$ and Schrötter. ${ }^{3}$ I have been unable to find any record of an erythema, though the "malar flush" occurring on the same side as the affected lung has been described by several writers. ${ }^{4}$ Parotitis is a very rare complication. In 5738 cases recorded in Vienna by Fismer it occurred only once. Only four cases have been recorded in Finglish medical literature. It is said to be most common in pneumonia occurring in the aged and most of the recorded cases were fatal. So far I have not been able to find many recorded cases of pneumococcal abscess and none occurring apart from cases in which a pnenmococcal arthritis existed as well. Two of the latter have been described by Nathan Raw, ${ }^{5}$ one occurring in the abdominal wall and the other in the thigh. A friend of mine had one in the abdominal wall occurring in his private practice some years ago and though this case was not verified bacteriologically the clinical history was very similar to the others described.

I am indebted to Dr. J. O. Muir, medical superintendent, for permission to publish these cases.

Fulham-road, S.W.

\section{A CASE OF}

\section{PRIMARY CANCER OF LEFT BRONCHUS,} WITH UNUSUAL ASSOCIATION OF PRESSURE SYMPTOMS; SECONDARY GROWTHS IN THYROID AND LYMPHATIC GLANDS. ${ }^{1}$

BY GEORGE A. ALLAN, M.B. GLASG.,

TUTOR IN CLINICAL MEDICINE, WESTERN INFIRMARY; ASSISTAYT TO THE PROFESSOR OF PRACTICE OF MEDICINE, ANDERSON'S COLLEGE, GLASGOW.

THIs case seemed worth recording not only on account of the comparative rarity of the pathological condition but also because of the somewhat unusual association of pressure symptoms which developed in the course of the disease.

The patient, a man, aged 38 years, was admitted to the Western Infirmary, Glasgow, on June 20th, 1906, under the care of the late Dr. James Finlayson, whose resident assistant I was at that time. His complaint was of pains in the left side of the chest, above the clavicle and in the left arm, and also of shortness of breath, all of about two months' duration. In January of the same year he had been treated for "pneumonia with pleurisy" of the left lung. This illness began with chill and was characterised by stabbing pain in the side, cough, and expectoration of "thick, sticky, brownish-red material." He was in bed four weeks and all his symptoms disappeared and he returned to work two weeks later. He remained at his work and was free of all symptoms for over a month, but about the end of April he began to suffer from pains which developed in the lateral region of the left chest and extended to the front and towards the left clavicle. Then the pain extended to the left shoulder, shooting down toward the elbow, and sometimes was so severe as to prevent him using the limb. He continued at work till early in June, notwithstanding the severity of the pain, though he was occasionally laid up for a day or two. Other symptoms had now supervened. He had severe paroxysmal cough coming on at short intervals, with a frothy white or yellowish expectoration, and the fits of coughing were frequently associated with vomiting and he also suffered from breathlessness on exertion, general weakness, hoarse and weak voice, and loss of appetite, flesh, and colour. His loss of wejght was over two stones since the beginning of his illness. Prior to the illness in January his health had been good but for tonsillitis in earlier years. There was no history of injury to the chest or of syphilis. He took alcohol daily in varying amount. His family history was vague, but probably his mother died from malignant disease in the abdomen.

1 Gerhardt's Handbuch, Band iii., S. 657. Symptomen, p. 65 .

3 Sitzungsbericht der Wiener Academie, Band lviii., S. 57 4 Wilson Fox: Diseases of Lungs.

1 A paper read at a meeting of the Glasgow Medico-Chirurgical Society on May 3rd, 1907.
On admission to hospital his temperature was $98.6^{\circ} \mathrm{F}$. his pulse was 108 , and his respirations were 32 per minute. He was moderately emaciated, with wasted limbs, hollow cheeks, sunken eyes, and the face presented a general sallow appearance with dilated capillaries over the malars. There were pigmented areas on the sides of the face and neck, with pale patches between, the resulting appearance suggesting vitiligo. He was weak and easily became breathless and had a spasmodic cough with muco-purulent expectoration showing no trace of blood. His voice was husky but weak. There was distinct fulness of the chest on the left side, both in the supra- and infra-clavicular regions, especially toward the sternum. The intercostal spaces, while visible on the right side, were filled ont on the left, and dilated veins ramified over the surface of this area, which was also pigmented. There was no pulsation in this region. At the level of the nipple the left side measured 17 inches and the right side $174_{4}^{3}$ inches. The right lung appeared to be normal on physical examination. Expansion of the left side of the chest was practically absent. On percussion there was absolute dulness in front from the level of the second rib downwards and behind from the level of the third dorsal spine downwards. Above the second rib in front resonance was "Skodaic" in quality. Over the dull area vocal fremitus and resonance were absent, as was also the respiratory murmur. The heart's sounds were, however, well conducted over this area. No râles were heard. The left arm was swollen as compared with the right, although the patient was right-handed. Above the left clavicle a hard mass could be felt on the deep surface of the sterno-mastoid muscle at its origin and further out enlarged and indurated glands could be isolated above and on the clavicle. Enlarged lymphatic glands were also felt in the left axilla along the axillary vessels. Nothing abnormal was noted in the circulatory or urinary systems. The appetite was poor and the tongue was coated. There was vomiting, sometimes accompanied by coughing, but there was no dysphagia. Physical examination of the abdomen for a tumour was negative. The pupils were unequal, the left being contracted, responding only slightly to light or on convergence. Movements of the eyeballs were unimpaired. There was slight ptosis of the left eyelid. The left vocal cord was fixed in the cadaveric position while the movements of the right cord were free and it crossed the middle line on phonation. The knee-jerks were present but not exaggerated. The patient was under observation in the wards for three months and becarne progressively weaker. He had frequent attacks of dyspncea, and breathing at all times was carried on with some degree of difficulty. The temperature was remittent in type throughout, there being a daily range of about $2 \frac{1}{2}^{\circ} \mathrm{F}$. The afternoon temperature reached about $102^{\circ}$ on an average-the maximum being $103^{\circ} 4^{\circ}$ and the lowest morning reading was $98^{\circ}$. Expectoration was never abundant and was muco-purulent in character and never at any time was it observed to contain blood. Various organisms were present in it but no tubercle bacilli or any cancer tissue. The swelling of the left arm increased and during the later weeks pain in the shoulder and down the arm was the chief complaint, for which morphine was regularly administered. The pain in the right side which developed three weeks before death was found to be due to pleurisy, and friction was detected. The pain disappeared, and as after this time the patient was exceedingly weak and made no particular complaint a detailed examination was not made and so a pleural effusion on the right side was overlooked. Physical signs on the left side remained practically unaltered throughout, but râles were detected on rare occasions. Within a week or so before death local hyperidrosis developed, affecting only the right side of the head and face, that is on the opposite side to the pulmonary lesion. The perspiration was so profuse while it lasted that the sweat dropped from his hair and face on to his shoulder. This was noted on two or three occasions. Generalised sweating had been a feature during the illness but it was only at these times that the local form was seen. He died quietly on Sept. 25th, 1906, five months after the onset of definite symptoms and eight months after the " pneumonia."

Necropsy. - The left lung was strongly adherent to the ribs, filling the thorax and extending beyond the middle line, while the heart was slightly displaced to the right side. There was a great deal of diffuse hardness in the anterior mediastinum, the connective tissue in which was found to be cedematous, harder and more fibrous than usual. The right 